\title{
Additional constraints in adsorption-desorption kinetics
}

\author{
S. J. Manzi, ${ }^{*}$ R. E. Belardinelli, G. Costanza, and V. D. Pereyra \\ Departamento de Física, Instituto de Física Aplicada (INFAP)_CONICET, Universidad Nacional de San Luis, Chacabuco 917, \\ 5700 San Luis, Argentina
}

(Received 2 June 2008; published 5 February 2009)

\begin{abstract}
In this work, the adsorption-desorption kinetic in the framework of the lattice gas model is analyzed. The transition probabilities are written as an expansion of the occupation configurations. Due to that, the detail balance principle determine half of the adsorption $A_{i}$ and desorption $D_{i}$ coefficients, consequently, different functional relations between them are proposed. Introducing additional constrains, it is demonstrated that when those coefficients are linearly related through a parameter $\gamma$, there are values of lateral interaction $V$, that lead to anomalous behavior in the adsorption isotherms, the sticking coefficient and the thermal programmed desorption spectra. Diagrams for the allowed values of $V$ and $\gamma$ are also shown. Alternatively, a more reliable formulation for the adsorption desorption kinetic based on the transition state theory is introduced. In such way the equilibrium and non equilibrium observables do not present anomalous or inconsistent behavior.
\end{abstract}

DOI: 10.1103/PhysRevE.79.021103

PACS number(s): 02.50.-r, 68.43.Mn, 68.43.Nr, 47.11.Qr

\section{INTRODUCTION}

The importance of surfaces and interfaces in a wide variety of fields has inspired an enormous interest in their structure and dynamics. In particular, the study of the kinetic and dynamic of the surface processes is of fundamental interest in the understanding of heterogeneous catalysis and other processes taking place in gas-solid interfaces [1-14]. The kinetic is fundamentally determined by the energy transfer [15].

The rate of change in the adsorbate can be written as a difference between adsorption and desorption terms. This can be made in a heuristic way or in the framework of a rigorous approach using the nonequilibrium thermodynamic theory $[7,15,16]$. If during desorption the adsorbate does not remain in quasiequilibrium or, more specifically, the adsorbate configurations are not close to those predicted by the grand canonical distribution, the description of the system through macroscopic variables is not enough and it should be carried out based on the nonequilibrium statistical mechanics that involves time-dependent distribution functions.

The kinetic lattice gas model (KLGM) is an example of such theory, and it was set up in close analogy to the timedependent Ising model for magnetic systems, which was originally introduced by Glauber $[17,18]$. In its simplest form, the KLGM is restricted to the submonolayer regime and to the gas-solid system where the surface structure and the adsorption sites do not change with the coverage [19]. However, further generalizations of the lattice-gas model have been made in the past. For example, the multilayer lattice gas model was used for analyzing and simulating desorption data for $\mathrm{Li}$ and $\mathrm{Cs}$ on $\mathrm{Ru}(0001)$ [20,21], the influence of the precursor state in the adsorption-desorption kinetic [16] and other related problems [22], the adsorptiondesorption kinetic with multiple site occupations [23-25], etc.

On the other hand, it is well known that different microscopic dynamics can yield different equilibrium paths and

\footnotetext{
*smanzi@unsl.edu.ar
}

equilibrium fluctuations [26] (cluster versus local MC algorithms being the most extreme example [27]) and even noticeable differences in the steady-state microstructure $[28,29]$. Recent studies indicate that different stochastic dynamics, even when they have the same conserved quantities and satisfy the detailed balance principle, lead to important differences in the nanostructure of field-driven interfaces [30-34].

Before defining the transition probabilities, an important distinction must be made. That is, between models with hard dynamics [32,34-39], in which the single-site transition rates cannot be factorized into one term that depends only on the interaction energies, in contrast with those models with soft dynamics $[31,33,34,40-46]$, for which this factorization is possible.

In this context, Kang et al. [44] have analyzed different choices for the transition probabilities in MC simulations for studying the growth exponent in the growth of domains. They showed that the choice of transition probabilities affects directly the dynamic quantities. Particularly, they found this dependence in the growth exponent in the ordered domains out of equilibrium [47]. In the same way, Rikvold et $a l$. have shown that the intrinsic interface width and properties in field-driven solid-on-solid interface studies depend on the choice of the dynamics. They have found that, in the framework of the soft dynamics, all dependence on the field canceled due to the detailed balance principle [31,33,34]. While in hard dynamics the intrinsic interface width and properties, such as the propagation velocity, are strongly affected by the field driven [31].

Despite the fact that detailed microscopic mechanisms of the surface processes are usually not known, in the KLGM the transition probabilities can be written in terms of the occupation configurations of all neighboring sites $[7,15,16,19,22,48-50]$. In this point, the detailed balance principle imposes a set of restrictions on the coefficients of adsorption $A_{i}$, desorption $D_{i}$, diffusion $C_{i}$, etc., as is discussed by Kreuzer and co-workers [15,16,19,22], where the authors have introduced different kinetics according to the relations among those coefficients. In fact, any functional relation between the coefficients in the transition probabili- 
ties must be postulated ad hoc, or calculated from microscopic Hamiltonian that account for coupling of the adsorbate to the lattice or electronic degrees of freedom of the substrate, for example, by using the transition state theory (TST) $[51,52]$. This theory, worked out by Eyring, Wigner, and Pelzer about 70 years ago, forms the mainstay of the gas- and liquid-phase kinetics (for the current status of TST and numerous references, see a recent paper by Schenter et al. [53]). In the framework of the TST, the adsorption and desorption kinetic and other rate processes were extensively analyzed in the past, particularly in a series of paper written by Zhdanov (see Refs. [12,54-59]).

On the other hand, when adsorption and desorption are the only processes taken into account, Kreuzer and coworkers have assumed a linear relation $A_{i}=\gamma D_{i}$ (where $\gamma$ is a proportionality parameter). In such a way the authors have obtained the adsorption-desorption kinetic calculating the sticking coefficient for different cases, including Langmuir kinetic, sticking on random adsorbate and the influence of intrinsic and extrinsic precursors [16]. Moreover, very recently, Payne and Kreuzer have discussed the one and twodimensional diffusion with different kinetic schemes, using the same methodology $[48,49]$.

Although those coefficients satisfy the detailed balance principle, the relation between them depends strongly on the lateral interaction energy and must be carefully analyzed in order to describe the adsorption and desorption processes, otherwise the results could lead to anomalous general behaviors in the kinetics. The aim of the present paper is to demonstrate that, even for the simplest one-dimensional case, a linear relation among the adsorption and desorption coefficients gives some inconsistencies. With this purpose, the adsorption desorption kinetic is analyzed for a 1D KLGM with nearest-neighbor lateral interaction $V$.

The analytical treatment has been done in a onedimensional system, mainly due to two reasons. The first one is the availability of the exact solution for the coverage and two-sites correlation function (which is not possible in higher dimensions). The second one is the presence of phase transitions in two and higher dimensions. This could mask the possible anomalous behaviors of the observables, which is precisely the objective of the present investigation.

The outline of the paper is as follows. In Sec. II, the KLGM is setup, introducing the master equation and writing the transition probabilities in terms of the occupation configurations of all neighboring sites. A set of restrictions on the adsorption and desorption coefficients are obtained according to the detailed balance principle. In order to fix the unknown coefficients in the transition probabilities, two different linear relations are proposed. The equilibrium and non equilibrium properties of the system are analyzed solving the rate equations for the coverage and higher correlations. Additional inequalities among the adsorption and desorption parameters are found, determining forbidden values for the parameters $\gamma$ and $V$, for which, the adsorption isotherms, sticking coefficients and thermal desorption spectra are ill behaved. Diagrams with forbidden regions as function of lateral interaction $V$ and the parameter $\gamma$ are shown. In Sec. IV, the transition state theory is introduced to obtain the adsorption desorption kinetic. Finally, in Sec. V our conclusions are presented.

\section{THE KINETIC LATTICE GAS MODEL AND THE MASTER EQUATION}

To set up the KLGM one restricts the analysis to a gassolid system in which all relevant processes, such as diffusion, adsorption, desorption, reactions, etc., are Markovian. One assumes that the system can be divided into cells, labeled $i$, for which one introduces microscopic variables $n_{i}$ $=1$ or 0 depending on whether cell $i$ is occupied by an adsorbed gas particle or not. The connection with magnetic systems is made by a transformation to spin variables $\sigma_{i}$ $=2 n_{i}-1$. To introduce the dynamic of the system one writes down a model Hamiltonian

$$
H=E_{s} \sum_{i} n_{i}+\frac{1}{2} V \sum_{\langle i j\rangle} n_{i} n_{j}+\cdots .
$$

Here $E_{s}$ is a single particle energy, $V$ is the two particle interaction between nearest neighbors $\langle i j\rangle$. Interactions between next-nearest neighbors, etc., and many particle interactions can be easily added to Eq. (1).

As long as the number of particles in the adsorbate does not changed, which is the case for systems in equilibrium or diffusion studies, the first term in Eq. (1) is constant and can be dropped from further consideration. However, if the objective is the study of the adsorption-desorption kinetic, the number of particles in the adsorbate changes as a function of time and a proper identification of $E_{s}$ is mandatory. Arguing that the lattice gas Hamiltonian should give the same Helmholtz free energy as a microscopic Hamiltonian (for noninteracting particles) one can show that the proper identification is given by [60]

$$
E_{s}=-V_{0}-\frac{1}{\beta} \ln \left(q_{3} q_{\text {int }}\right)-\frac{1}{\beta}\left[\ln \left(\beta \lambda^{3} P\right)-\ln \left(Z_{\text {int }}\right)\right],
$$

where $\beta=1 / k_{B} T ; k_{B}$ and $T$ are the Boltzmann constant and the absolute temperature, respectively. $V_{0}$ is the (positive) depth of the surface potential, $q_{3}$ is the single particle partition function of an adsorbed particle, $q_{\text {int }}$ is the internal partition function for (frustrated) vibrations and rotations of the adsorbed molecule; $P$ is the pressure in the gas phase above the surface and $\lambda=h / \sqrt{2 \pi m k_{B} T}$ is the thermal wavelength of the adparticle with mass $m$, the partition function of which for (free) vibrations and rotations is $Z_{\text {int }}$.

One introduces a function $P(\mathbf{n}, t)$ which gives the probability that a given microscopic configuration $\mathbf{n}$ $=\left(n_{1}, n_{2}, \ldots, n_{M}\right)$ is realized at time $t$, where $M$ is the total number of adsorption sites on the surface. It satisfies a master equation

$$
\frac{d P(\mathbf{n} ; t)}{d t}=\sum_{\mathbf{n}^{\prime}}\left[W\left(\mathbf{n} ; \mathbf{n}^{\prime}\right) P\left(\mathbf{n}^{\prime} ; t\right)-W\left(\mathbf{n}^{\prime} ; \mathbf{n}\right) P(\mathbf{n} ; t)\right]
$$

where $W\left(\mathbf{n}^{\prime} ; \mathbf{n}\right)$ is the transition probability that the microstate $\mathbf{n}$ changes into $\mathbf{n}^{\prime}$ per unit time. It satisfies detailed balance

$$
W\left(\mathbf{n}^{\prime} ; \mathbf{n}\right) P_{0}(\mathbf{n})=W\left(\mathbf{n} ; \mathbf{n}^{\prime}\right) P_{0}\left(\mathbf{n}^{\prime}\right),
$$

where 


$$
P_{0}(\mathbf{n})=Z^{-1} \exp [-\beta H(\mathbf{n})]
$$

is the equilibrium probability and $Z$ is the partition function given by

$$
Z=\sum_{\{\mathbf{n}\}} e^{-\beta H(\mathbf{n})} .
$$

In principle, $W\left(\mathbf{n}^{\prime} ; \mathbf{n}\right)$ must be calculated from a Hamiltonian that includes, in addition to Eq. (1), coupling terms to the gas phase and the solid that mediate mass and energy exchange. However, depending of the system, different expressions for transition probabilities can be proposed. In transition dynamic approximation $[41,45]$, transition rates cannot be factorized into one part that depends only on the interaction energy and another that depends only on the field energy [34] (hard dynamic [35]).

Usually, one follows the procedure introduced by Glauber and guesses an appropriate form for $W\left(\mathbf{n}^{\prime} ; \mathbf{n}\right)$. One further assumes that the duration of an individual transition, e.g., hopping to a neighboring site, is much shorter than the residence time in the initial state. In this situation there will be only one transition at any given time and the total transition probability as a sum of individual terms can be written.

In order to analyze the simplest case, let us consider the one-dimensional lattice gas with nearest-neighbor interactions where only direct adsorption and desorption processes are taken into account (no other processes are considered), in this case, the transition probability can be written as

$$
\begin{aligned}
W_{\text {ad-des }}\left(\mathbf{n}^{\prime} ; \mathbf{n}\right)= & \sum_{i}\left\{w _ { a } ( 1 - n _ { i } ) \left(A_{0}+A_{1}\left(n_{i-1}+n_{i+1}\right)\right.\right. \\
& \left.+A_{2} n_{i-1} n_{i+1}\right)+w_{d} n_{i}\left[D_{0}+D_{1}\left(n_{i-1}+n_{i+1}\right)\right. \\
& \left.\left.+D_{2} n_{i-1} n_{i+1}\right]\right\} \delta\left(n_{i}^{\prime}, 1-n_{i}\right) \Pi_{j \neq i} \delta\left(n_{j}^{\prime}, n_{j}\right)
\end{aligned}
$$

Here adsorption into site $i$ occurs if $n_{i}=0$ initially, with a rate controlled by prospective neighbors if $A_{i} \neq 0$. The Kronecker $\delta$ for sites $j \neq i$ excludes multiple transitions.

The motion equation for coverage can be obtained by multiplying the master equation by occupation number $n_{i}$ and summing overall sites. Thus one can obtain the following expression for the coverage $[8-10,23,50,61]$ :

$$
\begin{aligned}
\frac{d \theta}{d t}= & \left.w_{a}\left[A_{0}\langle\bigcirc\rangle+2 A_{1}\langle\bigcirc\rangle\right\rangle+A_{2}\langle\bigcirc \bigcirc\rangle\right] \\
& -w_{d}\left[D_{0}\langle\mathbf{\bigcirc}\rangle+2 D_{1}\langle\bigcirc\rangle+D_{2}\langle\bigcirc \bigcirc\rangle\right] .
\end{aligned}
$$

$\langle\bigcirc\rangle,\langle\bigcirc \bigcirc\rangle,\langle\bigcirc \bigcirc\rangle\rangle,\langle\bigcirc\rangle,\langle\bigcirc\rangle\rangle$, and $\langle\bigcirc \bigcirc\rangle\rangle$ symbols represent correlation functions. Here and elsewhere, some intermediate factorizations and manipulations of correlators are conveniently carried out in diagrammatic form. For example, $\langle\bigcirc\rangle$ denotes the average occupation number for the second order moment which evaluates the probability that the site located at the right of an occupied site would be empty. With the same procedure the rate equations for the next correlation functions for three independent sites can be written as

$$
\begin{aligned}
& \frac{d\langle\bigcirc\rangle}{d t}=2 w_{a}\left[\left(A_{0}+A_{1}\right)\langle\bigcirc \bigcirc\rangle+\left(A_{1}+A_{2}\right)\langle\bigcirc \bigcirc\rangle\right] \\
& -2 w_{d}\left[\left(D_{0}+D_{1}\right)\langle\bigcirc\rangle+\left(D_{1}+D_{2}\right)\langle\bigcirc \bigcirc\rangle\right],
\end{aligned}
$$

$$
\begin{aligned}
\frac{d\langle\bigcirc \bigcirc\rangle}{d t}= & w_{a}\left[2 A_{0}\langle\bigcirc \bigcirc \bigcirc\rangle+2 A_{1}\langle\bigcirc \bigcirc \bigcirc \bigcirc\rangle\right. \\
& \left.-\left(A_{0}+2 A_{1}+A_{2}\right)\langle\bigcirc \bigcirc \bigcirc\rangle\right] \\
& -w_{d}\left[2 D_{0}\langle\bigcirc \bigcirc \bigcirc\rangle+2 D_{1}\langle\bigcirc \bigcirc \bigcirc \bigcirc\rangle\right. \\
& \left.-\left(D_{0}+2 D_{1}+D_{2}\right)\langle\bigcirc \bigcirc \bigcirc\rangle\right]
\end{aligned}
$$

$$
\begin{aligned}
& \frac{d\langle\bigcirc \bigcirc\rangle}{d t}=w_{a}\left[2\left(A_{0}+A_{1}\right)\langle\bigcirc \bigcirc \bigcirc\rangle+\left(A_{0}+2 A_{1}+A_{2}\right)\langle\bigcirc \bigcirc \bigcirc\rangle\right. \\
& \left.+2\left(A_{1}+A_{2}\right)\langle\bigcirc \bigcirc \bigcirc\rangle\right] \\
& -w_{d}\left[\left(3 D_{0}+4 D_{1}+D_{2}\right)\langle\bigcirc \bigcirc\rangle\right. \\
& \left.\left.+2\left(D_{1}+D_{2}\right)\langle\odot \bigcirc)\right\rangle\right] \text {. }
\end{aligned}
$$

The detailed balance principle imposes a set of restrictions on the coefficients $A_{i}$ and $D_{i}$ [22] are

$$
\begin{gathered}
w_{a} A_{0}=w_{d} D_{0} e^{-\beta E_{s},} \\
w_{a}\left(A_{0}+A_{1}\right)=w_{d}\left(D_{0}+D_{1}\right) e^{-\beta\left(E_{s}+V\right)},
\end{gathered}
$$

and

$$
w_{a}\left(A_{0}+2 A_{1}+A_{2}\right)=w_{d}\left(D_{0}+2 D_{1}+D_{2}\right) e^{-\beta\left(E_{s}+2 V\right)} .
$$

Note that in general $w_{a}$ and $w_{d}$ cannot be fixed by detailed balance because they contain the information about the energy exchange with the solid in the adsorption and desorption processes not present in the static lattice gas Hamiltonian [22]. However, if one considers that $w_{a}=w_{d}=w_{0}$, and by comparison with the phenomenological expression for adsorption, one can identify it as

$$
w_{0}=\frac{P \lambda a_{s}}{h},
$$

where $a_{s}$ is the area of a surface unit cell. It contains the dynamic information about the energy transfer from the adsorbing particle to the solid which gives rise to its temperature dependence, for instance, an exponential Boltzmann factor for activated adsorption. It can be calculated only on the basis of a dynamic theory that accounts for the coupling of the ad-particles to the vibrational and electronic degrees of freedom of the substrate, and must be postulated ad hoc within the context of the kinetic lattice gas model.

The long-range interaction introduces similar constraints on the other coefficients. Each such constraints introduces two new coefficients. However, detailed balance provides only half the number of relations to fix these unknown coefficients in the transition probabilities. Again, the static (lattice gas) Hamiltonian cannot completely dictate the kind of kinetic possible in the system. As it is pointed out in Refs. $[15,19,22]$, any functional relation between the $A$ and $D$ coefficients must be postulated ad hoc, or calculated from a 
microscopic Hamiltonian that accounts for coupling of the adsorbate to the lattice or electronic degrees of freedom of the substrate. In the next section two different relations among the adsorption and desorption coefficients are analyzed.

\section{LINEAR RELATIONS BETWEEN ADSORPTION AND DESORPTION COEFFICIENTS}

A linear relationship between the $A_{i}$ and $D_{i}$ coefficients is proposed (case $K 1)$ as

$$
\frac{A_{i}}{A_{0}}=\gamma \frac{D_{i}}{D_{0}}
$$

for $i=1,2$, where $\gamma$ is a proportionality coefficient. Note that this linear relation was previously proposed in Refs. $[15,19,22]$, where $A_{0}=1$ has been considered. The detailed balance principle imposes

$$
D_{1}=D_{0}\left(\frac{e^{\beta V}-1}{1-\gamma e^{\beta V}}\right)
$$

and

$$
D_{2}=D_{0}\left(\frac{e^{2 \beta V}-1}{1-\gamma e^{2 \beta V}}\right)-2 D_{1} .
$$

Other possible choice of the parameters $A_{i}$ and $D_{i}$, which fulfill the detailed balance and leads to a new formulation of the adsorption-desorption kinetic is (case $K 2$ )

$$
A_{2}=\gamma A_{1}
$$

and

$$
D_{2}=\gamma D_{1} .
$$

Considering only nearest-neighbor interactions one can replace Eqs. (19) and (20) in Eqs. (13) and (14) and find the following expressions for the coefficients:

$$
A_{1}=A_{0}\left(\frac{(2+\gamma) e^{-\beta V}-(1+\gamma) e^{-2 \beta V}-1}{(2+\gamma)\left(1-e^{-\beta V}\right)}\right)
$$

and

$$
D_{1}=D_{0}\left(\frac{(1+\gamma) e^{\beta V}-(2+\gamma)+e^{-\beta V}}{(2+\gamma)\left(1-e^{-\beta V}\right)}\right) .
$$

When adsorption and desorption coefficients are determined, the properties of the system such as adsorption isotherms, sticking coefficient and thermal desorption (TPD) spectra can be obtained.

However, to analyze correctly the consequences of the linear relations assumed above, let us introduce the following identities [62]:

$$
\langle\bigcirc\rangle=\langle\bigcirc \bigcirc\rangle+2\langle\bigcirc \bigcirc \bigcirc\rangle+\langle\bigcirc \bigcirc \bigcirc\rangle
$$

and

$$
\langle\bigcirc\rangle\rangle=\langle\bigcirc \bigcirc\rangle\rangle+\langle\bigcirc \bigcirc\rangle\rangle
$$

In such way, one can easily obtain alternative expressions for the equations (8)-(11), namely,

$$
\begin{aligned}
\frac{d \theta}{d t}= & w_{0}\left[\left(A_{0}\right)\langle\bigcirc \bigcirc \bigcirc\rangle+2\left(A_{0}+A_{1}\right)\langle\bigcirc \bigcirc \bigcirc\rangle\right. \\
& +\left(A_{0}+2 A_{1}+A_{2}\right)\langle\bigcirc \bigcirc \bigcirc]-w_{0}\left[\left(D_{0}\right)\langle\bigcirc \bigcirc \bigcirc\rangle\right. \\
& +2\left(D_{0}+D_{1}\right)\left\langle\bigcirc \bigcirc \bigcirc+\left(D_{0}+2 D_{1}+D_{2}\right)\langle\bigcirc \bigcirc\rangle\right],
\end{aligned}
$$

$$
\begin{aligned}
& \frac{d\langle\bigcirc\rangle}{d t}=2 w_{0}\left[\left(A_{0}+A_{1}\right)\langle\bigcirc \bigcirc \bigcirc\rangle+\left(A_{0}+2 A_{1}+A_{2}\right)\langle\bigcirc \bigcirc \bigcirc]\right. \\
& -2 w_{0}\left[\left(D_{0}+D_{1}\right)\langle\bigcirc \bigcirc \bigcirc\rangle\right. \\
& \left.+\left(D_{0}+2 D_{1}+D_{2}\right)\langle\odot \bigcirc\rangle\right] \\
& \frac{d\langle\bigcirc \bigcirc}{d t}=w_{0}\left[2 ( A _ { 0 } ) \left\langle\bigcirc \bigcirc \bigcirc \bigcirc+2\left(A_{0}+A_{1}\right)\langle\bigcirc \bigcirc \bigcirc\rangle\right.\right. \\
& -\left(A_{0}+2 A_{1}+A_{2}\right)\langle\bigcirc \bigcirc \bigcirc \\
& -\left(A_{0}+2 A_{1}+A_{2}\right)\langle\bigcirc \bigcirc \bigcirc \bigcirc] \\
& -w_{0}\left[2\left(D_{0}\right)\langle\bigcirc \bigcirc \bigcirc\rangle+2\left(D_{0}+D_{1}\right)\langle\bigcirc \bigcirc \bigcirc \bigcirc\right. \\
& -\left(D_{0}+2 D_{1}+D_{2}\right)\langle\bigcirc 0 \bigcirc \bigcirc\rangle \\
& \left.-\left(D_{0}+2 D_{1}+D_{2}\right)\langle\bigcirc \bigcirc \bigcirc\rangle\right] \text {, }
\end{aligned}
$$

$$
\begin{aligned}
& \frac{d\langle\boldsymbol{\bigcirc}\rangle}{d t}=w_{0}\left[2\left(A_{0}+A_{1}\right)\langle\bigcirc \bigcirc \bigcirc \bigcirc\rangle\right. \\
& +3\left(A_{0}+2 A_{1}+A_{2}\right)\langle\bigcirc \bigcirc\rangle \\
& \left.+\left(A_{0}+2 A_{1}+A_{2}\right)\langle\bigcirc \bigcirc \bigcirc\rangle\right] \\
& -w_{0}\left\{\left[2\left(D_{0}+D_{1}\right)+\left(D_{0}+2 D_{1}+D_{2}\right)\right]\langle\circlearrowright \bigcirc \bigcirc\rangle\right. \\
& \left.+3\left(D_{0}+2 D_{1}+D_{2}\right)\langle\bigcirc \bigcirc \bigcirc\rangle\right\} \text {. }
\end{aligned}
$$

Note that $\langle\bigcirc \bigcirc \bigcirc\rangle,\langle\bigcirc \bigcirc\rangle\rangle,\langle\bigcirc\rangle\rangle,\langle\bigcirc \bigcirc\rangle\rangle,\langle\bigcirc\rangle\rangle$, and

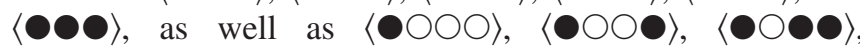

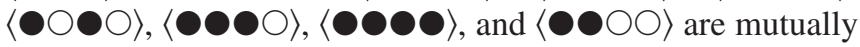
exclusive conditional probabilities, therefore each of the parentheses in Eqs. (25)-(28) must be positive. After some algebra one can obtain the following inequalities:

$$
\begin{gathered}
Q_{\mathrm{ads}}^{0}=A_{0} \geqslant 0, \\
Q_{\mathrm{ads}}^{1}=\left(A_{0}+A_{1}\right) \geqslant 0, \\
Q_{\mathrm{ads}}^{2}=\left(A_{0}+2 A_{1}+A_{2}\right) \geqslant 0, \\
Q_{\mathrm{des}}^{0}=D_{0} \geqslant 0, \\
Q_{\mathrm{des}}^{1}=\left(D_{0}+D_{1}\right) \geqslant 0,
\end{gathered}
$$

and

$$
Q_{\mathrm{des}}^{2}=\left(D_{0}+2 D_{1}+D_{2}\right) \geqslant 0 .
$$

It it easy to see that Eqs. (29)-(34) impose new restrictions on the $A_{i}$ and $D_{i}$ coefficients. If these additional restrictions are applied to the two proposed kinetics, certain values of $\gamma$ are not allowed. These forbidden values depend on the lateral interaction $V$ and they are shown in the diagrams of Fig. 1(a) 

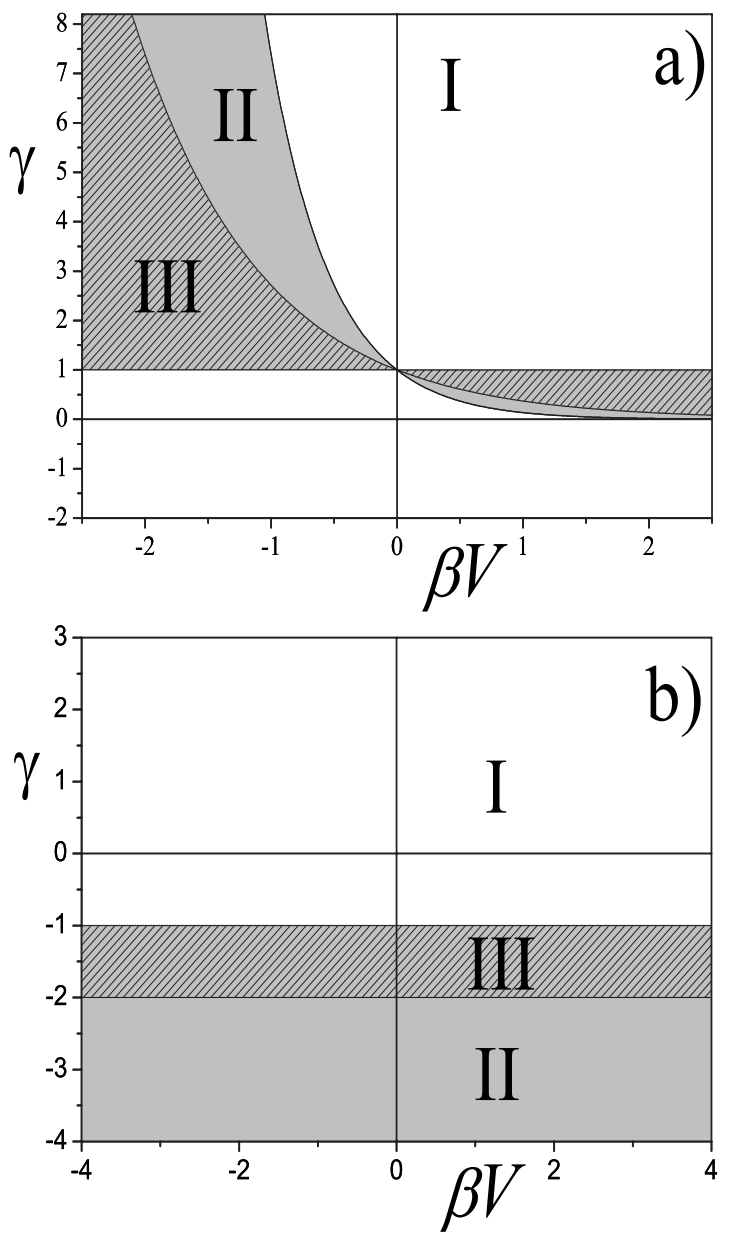

FIG. 1. Diagram $\gamma$ versus $\beta V$ corresponding to (a) $K 1$ and (b) $K 2$ proposed kinetics.

and 1(b) for the $K 1$ and $K 2$ kinetics, respectively.

Region I corresponds to those values of $\gamma$ and $V$ which make positive all the coefficients in Eqs. (29)-(34). Region II corresponds to those values of the parameters which make $Q_{\mathrm{ads}}^{1} \geqslant 0, Q_{\mathrm{des}}^{1} \geqslant 0, Q_{\mathrm{ads}}^{2}<0$, and $Q_{\mathrm{des}}^{2}<0$ (intermediate region). Region III corresponds to those values of $\gamma$ and $V$ which make all the coefficients negative (except $Q_{\text {ads }}^{0}$ and $Q_{\mathrm{des}}^{0}$ ), $Q_{\mathrm{ads}}^{1}<0, Q_{\mathrm{ads}}^{2}<0, Q_{\mathrm{des}}^{1}<0$, and $Q_{\mathrm{des}}^{2}<0$ (forbidden region).

To calculate the exact solution for adsorption isotherm the two first rate equations [Eqs. (25) and (26)] must be set equal to zero $[23,61]$. To calculate the sticking coefficient one uses the following definition [16]:

$$
S(\theta, T)=A_{0}\langle\bigcirc\rangle+2 A_{1}\langle\bullet \bigcirc\rangle+A_{2}\langle\bullet \bigcirc\rangle .
$$

The exact solution for TPD spectra of immobile particles is obtained by solving the rate equations for the first four correlations [Eqs. (25)-(28)], where the adsorption terms are neglected $[23,61,63]$. In all TPD spectra the desorption is considered as an activated process. Where the activation energy is $10 \mathrm{kcal} / \mathrm{mol}$, the pre-exponential factor is $10^{13} \mathrm{~s}^{-1}$ and the initial coverage is $\theta_{0}=0.9$.

The adsorption isotherms, sticking coefficients and TPD spectra for immobile adsorbate are shown in Figs. 2, 3, and
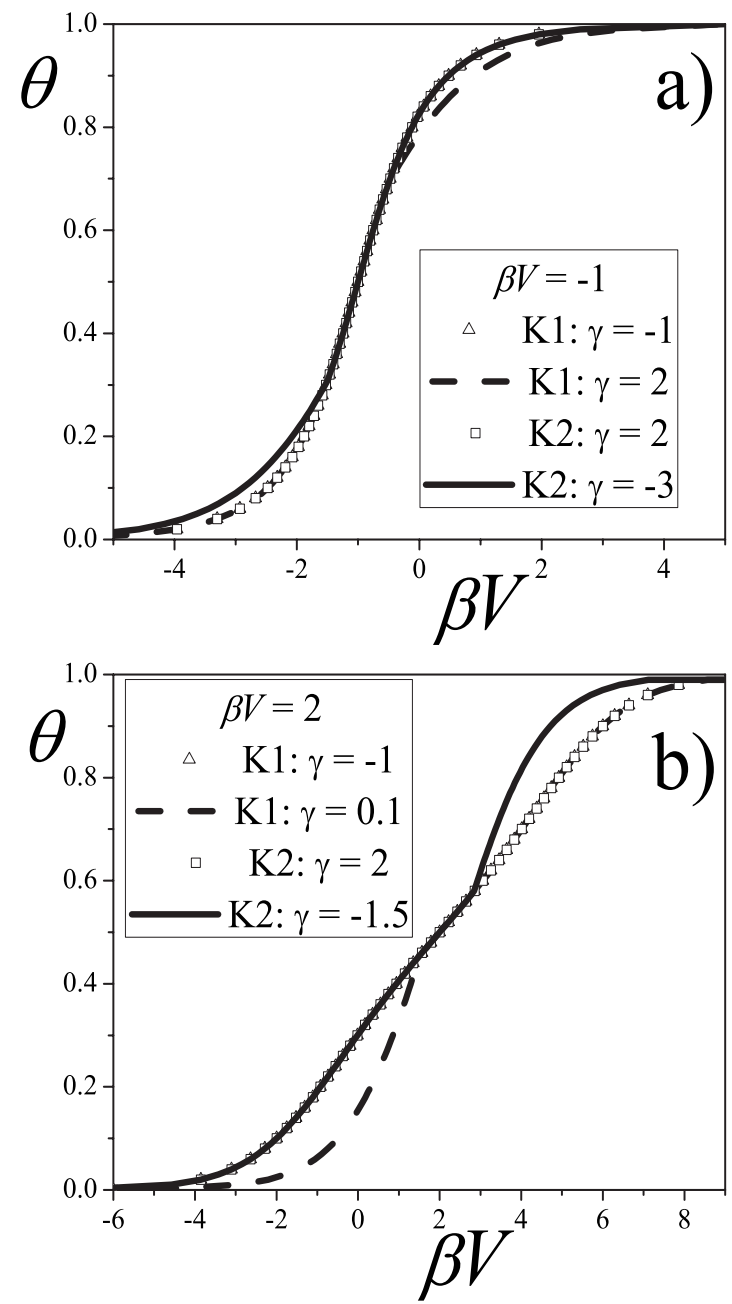

FIG. 2. Adsorption isotherms for different values of the parameter $\gamma$ and for two linear proposed kinetics. (a) Attractive and (b) repulsive lateral interaction.

4 , respectively. For those values of $\gamma$ out of the allowed region, the first derivative of the isotherm as a function of the chemical potential presents a discontinuity, and both the sticking coefficient and TPD spectra take negative values. These behaviors are certainly anomalous.

For values of $\gamma$ belonging to the allowed region the behavior of the observables do not present any inconsistencies. Although the physical meaning of the relations given in Eqs. (16), (19), and (20) is not clear, it seems to be perfectly valid in view that they fulfill the detailed balance. However, it must have certain coherence in the behavior of the observables according to the lateral interaction. For example, when repulsive lateral interaction is considered, the adsorption isotherms for monomers present a characteristic plateau at coverage $\theta=1 / 2$ for low enough temperature. This is due to an ordering of the adsorbed particles, namely, there is an alternation between particles and vacancies. For the same interaction the sticking coefficient will be below the straight line $S(\theta, T) / S_{0}(T)=1-\theta$, which corresponds to the Langmuir kinetic (null interaction) [16]. Finally, the immobile TPD for high initial coverages will have three peaks, as expected $[64,65]$. This behavior cannot be reproduced by using one of the proposals $K 1$ or $K 2$. 

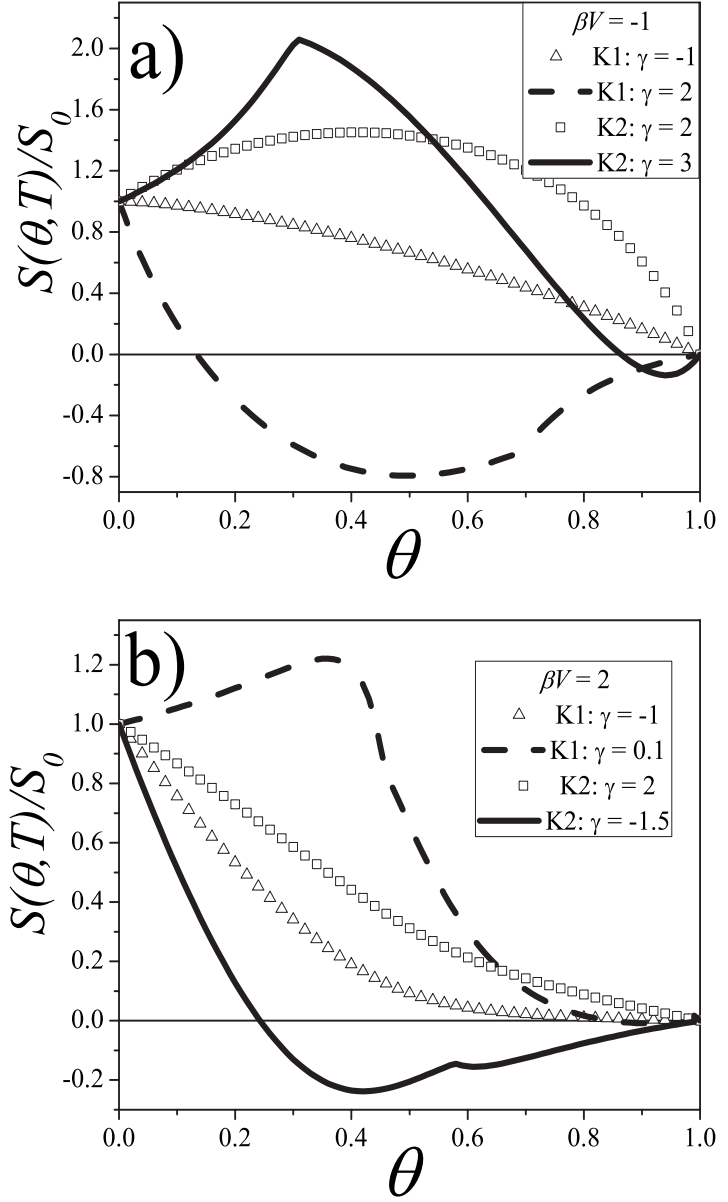

FIG. 3. Sticking coefficients for different values of the parameter $\gamma$ and for two linear proposed kinetics. (a) Attractive and (b) repulsive lateral interaction.

Moreover, in the framework of proposal $K 1$, the three peaks in the immobile TPD can be observed only in the limit $\gamma \rightarrow 0$. However, this value of $\gamma$ corresponds to the Langmuir kinetic.

As is discussed here the relation among the adsorption and desorption coefficients must be carefully chosen. In particular, linear relations between them, as is proposed by Kreuzer and co-workers in Refs. $[15,19,22]$ are not valid in general and some values of the parameter $\gamma$ and $V$ are not allowed. In fact, the observables obtained for those values of $\gamma$ and $V$ are ill behaved. Clearly, the detailed balance principle is not enough to guarantee the correct behavior of the kinetic and deeper analysis must be done to choose the functional relation between the $A$ and $D$ coefficients.

\section{TRANSITION STATE THEORY AND THE ADSORPTION AND DESORPTION COEFFICIENTS}

A theoretical calculation of the rate of an elementary physicochemical processes is a rather complicated procedure. This is true both for the description of the dynamic of the interaction on the atomic level and for statistical averaging over all the possible initial states of the system under investigation. Adsorption and desorption are examples of
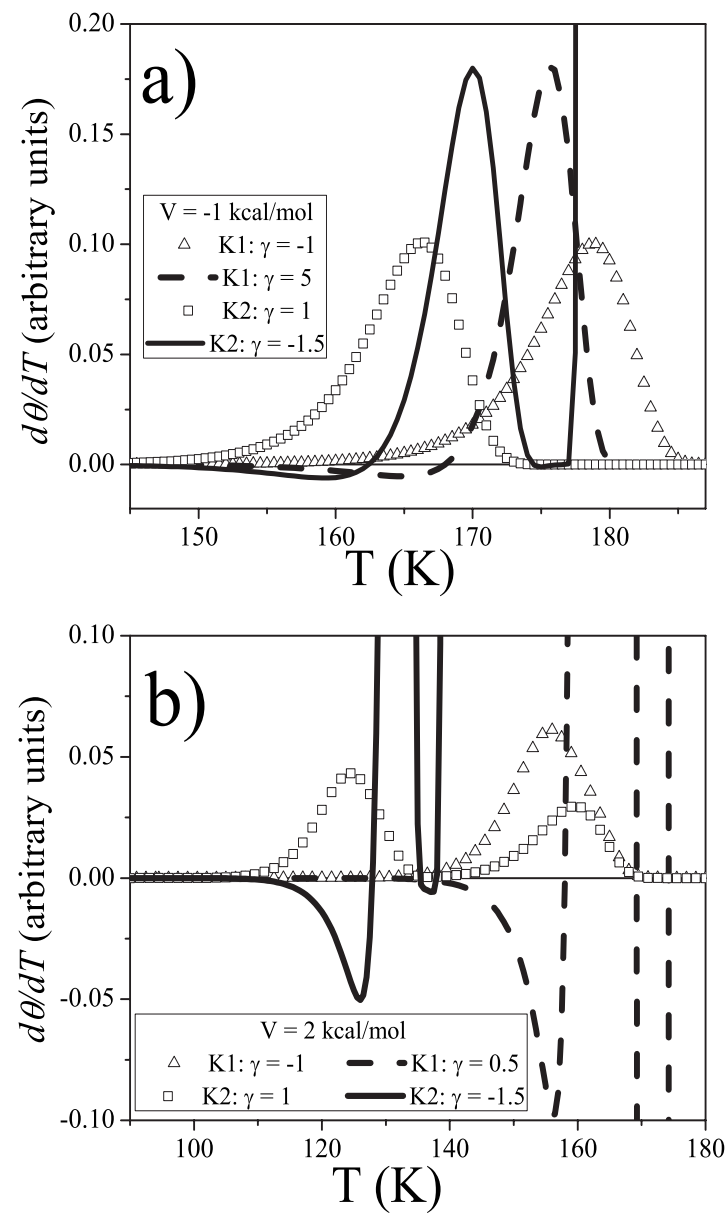

FIG. 4. Immobile TPD for different values of the parameter $\gamma$ and for two linear proposed kinetics. (a) Attractive and (b) repulsive lateral interaction.

physicochemical rate processes occurring at thermal conditions. The transition state theory provides a way to obtain the constant rates of the involved processes throughout the knowledge of the appropriate kinetic equation [12,54-59]. To describe the kinetic of monomolecular adsorption and desorption on uniform surface by using TST let us introduce the following expression for the coverage rate equation:

$$
\frac{d \theta}{d t}=k_{a} P-k_{d} \theta,
$$

where

$$
k_{a}=(1-\theta) k_{a}^{0} \sum_{i} P_{0, i} \exp \left(-\beta \epsilon_{i}^{*}\right)
$$

and

$$
k_{d}=k_{d}^{0} \sum_{i} P_{A, i} \exp \left[-\beta\left(\epsilon_{i}^{*}-\epsilon_{i}\right)\right]
$$

In these equations, $P_{0, i}$ is the conditional probability that a vacant site has the environment denoted by index $i$ and $P_{A, i}$ is a similar conditional probability for an adsorbed particle. In the framework of TST, $k_{a}^{0}$ and $k_{d}^{0}$ are the adsorption and desorption constants corresponding to the low-coverage 
limit; $\epsilon_{i}$ and $\epsilon_{i}^{*}$ are the lateral interactions (with neighbors) in the initial (ground) and activated (transition) states. These interactions can be calculated by using DFT [66-74]. Note that Eq. (36) is a purely phenomenological expression. Correlations can be included by using pair approximations, such as the quasichemical or even more sophisticated approximations [12]. Although in the case of the nearest-neighbor interactions the quasichemical approximation is accurate provided that surface diffusion is fast and the adsorbed overlayer is close to equilibrium, its applicability is limited in general. In fact, to obtain the exact solution for the immobile thermal desorption, one needs to solve the first four correlations [Eqs. (25)-(28)], where the adsorption terms are neglected $[23,61,63]$.

Density-functional theory calculations for a number of systems have shown that there are generally more than one interactions of appreciable magnitude between adsorbates. Analysis of the heat of adsorption as a function of coverage [72] phase diagrams of adlayers $[73,74]$, and other experiments point to the same fact.

In order to obtain additional information about the values of the adsorption and desorption coefficients, one can rewrite Eq. (36) considering a 1D system with nearest-neighbor interaction as

$$
\begin{aligned}
\frac{d \theta}{d t}= & P k_{a}^{0} e^{\beta E_{s} / 2}\left(\langle\bigcirc \bigcirc \bigcirc\rangle e^{-\beta\left(E_{s} / 2+\epsilon_{0}^{*}\right)}+2\langle\bigcirc \bigcirc \bigcirc\rangle e^{-\beta\left(E_{s} / 2+\epsilon_{1}^{*}\right)}\right. \\
& \left.\left.+\langle\bigcirc \bigcirc\rangle e^{-\beta\left(E_{s} / 2+\epsilon_{2}^{*}\right)}\right)-k_{d}^{0} e^{-\beta E_{s} / 2(\langle\bigcirc \bigcirc}\right\rangle e^{\beta\left(E_{s} / 2-\epsilon_{0}^{*}+\epsilon_{0}\right)} \\
& \left.+2\langle\bigcirc \bigcirc\rangle e^{\beta\left(E_{s} / 2-\epsilon_{1}^{*}+\epsilon_{1}\right)}+\langle\bigcirc \bigcirc\rangle e^{\beta\left(E_{s} / 2-\epsilon_{2}^{*}+\epsilon_{2}\right)}\right)
\end{aligned}
$$

In such way, one can identified the adsorption and desorption coefficients

$$
Q_{\mathrm{ads}}^{i}=\exp \left[-\beta\left(E_{s} / 2+\epsilon_{i}^{*}\right)\right]
$$

and

$$
Q_{\mathrm{des}}^{i}=\exp \left\{\beta\left[E_{s} / 2-\left(\epsilon_{i}^{*}-\epsilon_{i}\right)\right]\right\} .
$$

The detailed balance principle imposes that

$$
\epsilon_{i}=i V \text {. }
$$

The TST does not imposes any restriction on the value of the lateral interactions, either in the ground or activated states. Although the lateral interactions can be calculated by first principles methods, e.g., DFT, in some of the papers related to desorption kinetics $[65,75,76]$, the lateral interaction in the activated states is customarily believed to be weak compared to other interactions and neglected $\epsilon_{i}^{*}=0$. One of the reason is that at the UHV conditions the experimentalists tend to study systems where the sticking coefficient is high at all the coverages up to saturation. Clearly, this assumption means that the sticking coefficient corresponds to a Langmuir adsorption kinetics, independently on the value of $V$. However, in many situations important for practical catalysis, e.g., TPD in real systems (see Refs. $[77,78]$ ), or reaction kinetics $[79] \epsilon_{i}^{*} \neq 0$ are available as well. From physical considerations relations between both parameters $\epsilon_{i}$ and $\epsilon_{i}^{*}$ can be deduced. To this purpose, let us write the expression for sticking coefficient by using Eqs. (23), (24), and (39) as

$$
\begin{aligned}
& S(\theta, T)=S_{0}(T)\left[e^{-\beta \epsilon_{0}^{*}}\langle\bigcirc\rangle+2\left(e^{-\beta \epsilon_{1}^{*}}-e^{-\beta \epsilon_{0}^{*}}\right)\langle\bigcirc \bigcirc\rangle\right. \\
& \left.+\left(e^{-\beta \epsilon_{2}^{*}}-2 e^{-\beta \epsilon_{1}^{*}}+e^{-\beta \epsilon_{0}^{*}}\right)\langle\bigcirc \bigcirc\rangle\right] \text {. }
\end{aligned}
$$

The sticking coefficient at zero coverage is a function of temperature $S(\theta=0, T)=S_{0}(T)$ (see Refs. [80-82]), and is independent on any lateral interaction, then $\epsilon_{0}^{*}=0$. Therefore, Eq. (43) can be written as

$$
\begin{aligned}
S(\theta, T)= & S_{0}(T)\left[(1-\theta)+2\left(e^{-\beta \epsilon_{1}^{*}}-1\right)\langle\bigcirc \bigcirc\rangle\right. \\
& \left.+\left(e^{-\beta \epsilon_{2}^{*}}-2 e^{-\beta \epsilon_{1}^{*}}+1\right)\langle\bigcirc \bigcirc\rangle\right],
\end{aligned}
$$

where, from Eqs. (15) and (36)

$$
S_{0}(T)=\frac{k_{a}^{0} h}{\lambda a_{s}} .
$$

Assuming that the energy transfer in the adsorption process takes place within angstroms of the surface, the adsorbing particles actually experiences the interaction with particles already adsorbed on neighboring sites in more or less the same way as desorbing particles do, i.e., a lateral repulsion (attraction) will aid (hinder) desorption and conversely for adsorption as is discussed by many authors [16,22]. Therefore, it seems to be plausible that for attractive lateral interaction $(V<0)$ the next relation should be fulfilled:

$$
2\left(e^{-\beta \epsilon_{1}^{*}}-1\right)\left\langle\bigcirc \bigcirc+\left(e^{-\beta \epsilon_{2}^{*}}-2 e^{-\beta \epsilon_{1}^{*}}+1\right)\langle\bigcirc \bigcirc\rangle>0,\right.
$$

similarly, for repulsive lateral interaction $(V>0)$ :

$$
2\left(e^{-\beta \epsilon_{1}^{*}}-1\right)\left\langle\bigcirc \bigcirc+\left(e^{-\beta \epsilon_{2}^{*}}-2 e^{-\beta \epsilon_{1}^{*}}+1\right)\langle\bigcirc \bigcirc\rangle\langle 0 .\right.
$$

Equations (46) and (47) determine the allowed values for $\epsilon_{1}^{*}$ and $\epsilon_{2}^{*}$ as a function of coverage and ground state lateral interaction $V$. For $V=0$, one easily deduces that $\epsilon_{1}^{*}=0$ and $\epsilon_{2}^{*}=0$. From those equations, one also obtain that the lateral interaction in the activated state must have the same sign that the lateral interaction in the ground state.

Due to the fact that TST does not imposes any restriction on the value and sign of the lateral interaction, in both the ground and activated states, then several scenarios can be obtained for the desorption processes. Particularly, the presence of three peaks in the TPD curves of immobile particles is not exclusive of the repulsive lateral interactions. Moreover, one can obtain a TPD curve with three peaks, with both the activated and ground lateral interaction attractive, while the sticking coefficient for these interactions is higher than the Langmuirs sticking coefficient. Moreover, one can obtain a TPD spectra with only one peak for repulsive lateral interactions, which temperature corresponding to the peak is higher than the non-interacting case. These results are certainly unexpected.

In many cases, TST can be combined with the BrönstedPolanyi-type relations between the lateral interactions in the ground and activated states [83], for instance, 


$$
\epsilon_{i}^{*}=\frac{i V}{2}
$$

and an inverse relation between the $A$ and $D$ coefficients is found:

$$
\begin{gathered}
A_{0}=\frac{1}{D_{0}}, \\
A_{0}+A_{1}=\frac{1}{D_{0}+D_{1}},
\end{gathered}
$$

and

$$
A_{0}+2 A_{1}+A_{2}=\frac{1}{D_{0}+2 D_{1}+D_{2}} .
$$

After some algebra, one can easily obtain the following expressions for the $A$ and $D$ coefficients:

$$
\begin{gathered}
A_{0}=\exp \left[-\beta E_{s} / 2\right], \\
D_{0}=\exp \left[\beta E_{s} / 2\right], \\
A_{1}=\exp \left[-\beta E_{s} / 2\right](\exp [-\beta V / 2]-1), \\
D_{1}=\exp \left[\beta E_{s} / 2\right](\exp [\beta V / 2]-1), \\
A_{2}=\exp \left[-\beta E_{s} / 2\right](\exp [-\beta V / 2]-1)^{2},
\end{gathered}
$$

and

$$
D_{2}=\exp \left[\beta E_{s} / 2\right](\exp [\beta V / 2]-1)^{2} .
$$

In Figs. 5(a)-5(c), the adsorption isotherms, sticking coefficients and TPD spectra for different lateral interaction using the above formulation, are shown. As is observed, there are coherence between the behavior of the observables. In the framework of the TST, the observables obtained by using those values of the transition state lateral interaction that satisfies the inequalities given in Eqs. (46) and (47), do not present any anomalous behavior.

\section{SUMMARY AND CONCLUSIONS}

In this work, the adsorption-desorption kinetic in the framework of the one-dimensional lattice gas model with nearest-neighbor lateral interaction is considered. The master equation approach has been used to derive the rate equations for coverage and higher correlations, which give the equilibrium and nonequilibrium properties of the system. In order to obtain those equations, transition probabilities are written in terms of the occupation configurations. In such way, the detail balance principle imposes a set of restrictions on the adsorption $\left(A_{i}\right)$ and desorption $\left(D_{i}\right)$ coefficients. However, it determines half of them. To overcome such difficulty different functional relations among those coefficients can be postulated, the simplest one is a linear relation. However, it is observed that, when rate equations are written in terms of mutually exclusive conditional probabilities, additional constrains appear on $A_{i}$ and $D_{i}$ coefficients. These are rather
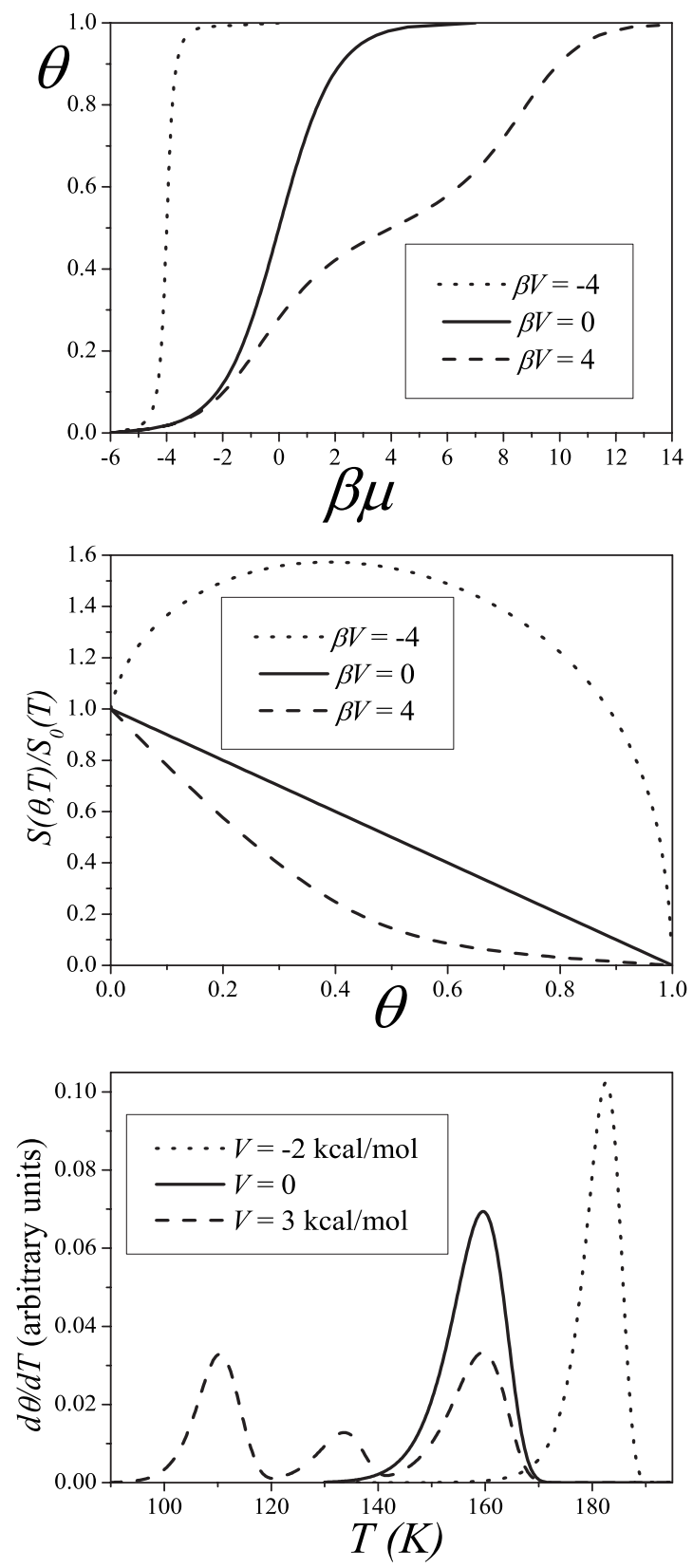

FIG. 5. (a) Adsorption isotherms, (b) sticking coefficients, and (c) immobile TPD, for attractive (dot), repulsive (dash), and null (line) interaction, using the inverse relation $L 3$.

restrictive because not all the values of the lateral interactions $(V)$ and linear parameter $(\gamma)$ are allowed. Moreover, the kinetics obtained for some values of these parameters are wrong, or at least, the equilibrium and nonequilibrium observables present anomalous behaviors. The diagrams of the allowed and forbidden values as a function of the parameters $V$ and $\gamma$, as well as the adsorption isotherms, sticking coefficient and TPD spectra for two different linear relations are showed. Three well-defined regions emerge from these diagrams in both cases. (i) The allowed region, where the adsorption-desorption kinetics is well behaved, i.e., the isotherms, sticking coefficients and the TPD spectra are well behaved too. It is also observed that Langmuir $\left(A_{i}=0\right)$ and interaction kinetics $\left(A_{i}=-D_{i}\right)$ belong to this region. (ii) The 
intermediate region, where the first derivative of the adsorption isotherms is discontinuous. This result is not possible in a one-dimensional lattice gas with nearest-neighbor lateral interaction. (iii) The forbidden region, where the adsorption isotherms behave like in region II and sticking coefficients are negative.

A third formulation based on the TST is introduced. From physical considerations on the behavior of the sticking coefficient, one concludes that additional relations should be fulfilled by the lateral interactions in the activated states. In addition, that no restriction is imposed by the TST on the value and sign of the interactions, therefore several scenarios can be obtained in the desorption processes depending on these values. Some of them are unexpected, i.e., the presence of three peaks in the TPD curves of immobile adsorbate is not exclusive of the repulsive lateral interactions. Considering the lateral interaction in the transition state as half of the lateral interaction in the ground state (the standard BrönstedPolanyi relation) an inverse relation among adsorption and desorption coefficients is obtained.

As a general conclusion, the detailed balance principle is not enough to guarantee the correct behavior of the kinetic.
The linear relations among the adsorption and desorption coefficients are not general and some values of the $V$ and $\gamma$ are not allowed. TST seems to be more adequate to analysis the adsorption desorption kinetic. On the other hand, both linear relations as well the inverse relation belong to the so called soft dynamics, where transition probabilities factorize into a part due only to the change in the field energy and a part due only to the change in the interaction energy.

Finally, it is important to emphasize that, even when the treatment has been done in a one-dimensional system, the results seem to be general and they do not depend on the dimensionality. However, the extension to higher dimensions, the incorporation of next-nearest neighbor interactions, as well as of diffusion terms should be done in the future.

\section{ACKNOWLEDGMENTS}

We thank Professor G. Zgrablich and Dr. M.R. Gómez for reading the manuscript. This work was partially supported by CONICET (Argentina).
[1] A. Cassuto and D. A. King, Surf. Sci. 102, 388 (1981).

[2] A. Córdoba and M. C. Lemos, J. Chem. Phys. 99, 4821 (1993).

[3] J. W. Evans, Rev. Mod. Phys. 65, 1281 (1993), and references therein

[4] D. Menzel, in Interactions on Metal Surfaces, edited by R. Gomer (Springer-Verlag, Berlín, 1975), p. 102.

[5] Y. K. Tovbin, in Equilibria and Dynamics of Gas Adsorption on Heterogeneous Solid Surfaces, edited by W. Rudzinski, A. Steele, and G. Zgrablich, Vol. 104 of Studies in Surface Science and Catalysis (Elsevier, New York, 1997), and references therein.

[6] V. P. Zhdanov and B. Kasemo, Chem. Phys. 177, 519 (1993), and references therein.

[7] H. J. Kreuzer and Z. W. Gortel, Physisorption Kinetics (Springer-Verlag, Berlin, 1986).

[8] H. J. Kreuzer and S. H. Payne, Surf. Sci. 198, 235 (1988).

[9] H. J. Kreuzer and S. H. Payne, Surf. Sci. 200, L433 (1988).

[10] S. H. Payne and H. J. Kreuzer, Surf. Sci. 205, 153 (1988).

[11] C. Uebing and V. P. Zhdanov, J. Chem. Phys. 109, 3197 (1998).

[12] V. P. Zhdanov, Elementary Physicochemical Processes on Solid Surfaces (Plenum, New York, 1991), Chap. 4, and references therein.

[13] M. Silverberg and A. Ben-Shaul, Surf. Sci. 214, 17 (1989).

[14] S. J. Lombardo and A. T. Bell, Surf. Sci. Rep. 13, 1 (1991).

[15] H. J. Kreuzer and S. H. Payne, in Studies in Surfaces Science and Catalysis [5], and references.

[16] H. J. Kreuzer, J. Chem. Phys. 104, 9593 (1996).

[17] R. J. Glauber, J. Math. Phys. 4, 294 (1963).

[18] K. Kawasaki, in Phase Transitions and Critical Phenomena, edited by C. Domb and M. S. Green (Academic Press, New York, 1972), Vol. 2, p. 443.
[19] H. J. Kreuzer and J. Zhang, Appl. Phys. A 51, 183 (1990).

[20] S. H. Payne, H. A. McKay, H. J. Kreuzer, M. Gierer, H. Bludau, H. Over, and G. Ertl, Phys. Rev. B 54, 5073 (1996).

[21] S. H. Payne, G. Ledue, J. C. Michael, and H. J. Kreuzer, Surf. Sci. 512, 151 (2002).

[22] S. H. Payne, H. J. Kreuzer, M. Kinne, R. Denecke, and H.-P. Steinruck, Surf. Sci. 513, 174 (2002).

[23] G. Costanza, S. Manzi, and V. D. Pereyra, Surf. Sci. 524, 89 (2003).

[24] O. Furlong, S. Manzi, G. Costanza, and V. D. Pereyra, Physica A 339, 267 (2004).

[25] G. Costanza, S. Manzi, and V. D. Pereyra, Surf. Sci. 600, 3484 (2006).

[26] O. Schochet, K. Kassner, E. Ben-Jacob, S. B. Lipson, and H. Müller-Krumbhaar, Physica A 181, 136 (1992), and references therein.

[27] D. P. Landau and K. Binder, Monte Carlo Simulations in Statistical Physics (Cambridge University Press, Cambridge, 2000).

[28] M. Siegert and M. Plischke, Phys. Rev. E 50, 917 (1994).

[29] Y. Shim and D. P. Landau, Phys. Rev. E 64, 036110 (2001).

[30] P. A. Rikvold and M. Kolesik, J. Stat. Phys. 100, 377 (2000).

[31] P. A. Rikvold and M. Kolesik, J. Phys. A 35, L117 (2002).

[32] P. A. Rikvold and M. Kolesik, Phys. Rev. E 66, 066116 (2002).

[33] P. A. Rikvold and M. Kolesik, Phys. Rev. E 67, 066113 (2003).

[34] G. M. Buendía, P. A. Rikvold, and M. Kolesik, Phys. Rev. B 73, 045437 (2006); J. Mol. Struct.: THEOCHEM 769, 207 (2006).

[35] J. Marro and R. Dickman, Nonequilibrium Phase Transitions in Lattice Model (Cambridge University Press, Cambridge, 1999), Chap. 7. 
[36] S. Frank, D. E. Roberts, and P. A. Rikvold, J. Chem. Phys. 122, 064705 (2005).

[37] K. Saito, S. Takesue, and S. Miyashita, Phys. Rev. E 61, 2397 (2000).

[38] K. Park and M. A. Novotny, Comput. Phys. Commun. 147, 737 (2002)

[39] G. M. Buendía, P. A. Rikvold, M. Kolesik, K. Park, and M. A. Novotny, Phys. Rev. B 76, 045422 (2007).

[40] S. Frank and P. A. Rikvold, Surf. Sci. 600, 2470 (2006).

[41] G. M. Buendía, P. A. Rikvold, K. Park, and M. A. Novotny, J. Chem. Phys. 121, 4193 (2004).

[42] K. Park, P. A. Rikvold, Gloria M. Buendía, and M. A. Novotny, Phys. Rev. Lett. 92, 015701 (2004).

[43] K. A. Fichthorn and W. H. Weinberg, J. Chem. Phys. 95, 1090 (1991).

[44] H. C. Kang and W. H. Weinberg, J. Chem. Phys. 90, 2824 (1989).

[45] T. Ala-Nissila and S. C. Ying, Prog. Surf. Sci. 39, 227 (1992); T. Ala-Nissila, R. Ferrando, and S. C. Ying, Adv. Phys. 51, 949 (2002)

[46] T. Ala-Nissila, J. Kjoll, and S. C. Ying, Phys. Rev. B 46, 846 (1992).

[47] K. Kawasaki, Phys. Rev. 145, 224 (1966) 148, 375 (1966); 150, 285 (1966).

[48] S. H. Payne and H. J. Kreuzer, Phys. Rev. B 75, 115403 (2007).

[49] S. H. Payne and H. J. Kreuzer, Phys. Rev. B 77, 121403(R) (2008).

[50] A. Wierzbicki and H. J. Kreuzer, Surf. Sci. 257, 417 (1991).

[51] S. Glasstone, K. Laidler, and H. Eyring, The Theory of Rate Processes (McGraw-Hill, New York, 1941).

[52] A. P. J. Jansen, Phys. Rev. B 69, 035414 (2004).

[53] G. K. Schenter, B. C. Garrett, and D. G. Truhlar, J. Chem. Phys. 119, 5828 (2003).

[54] V. P. Zhdanov, Surf. Sci. 111, 63 (1981); 111, L662 (1981); 123, 106 (1982); 133, 469 (1983); 157, L384 (1985); 165, L31 (1986); 171, L461 (1986); 209, 523 (1989).

[55] V. P. Zhdanov, Surf. Sci. Rep. 12, 185 (1991) and reference therein.

[56] V. D. Pereyra and G. Zgrablich, Langmuir 6, 118 (1990).

[57] V. D. Pereyra, G. Zgrablich, and V. P. Zhdanov, Langmuir 6, 691 (1990).

[58] B. Li, C.-S. Zhang, V. P. Zhdanov, and P. R. Norton, Surf. Sci. 322, 373 (1995).

[59] V. P. Zhdanov and B. Kasemo, Surf. Sci. 412, 527 (1998).
[60] H. J. Kreuzer and S. H. Payne, in Computational Methods in Colloid and Interface Science (Dekker, New York, 1999).

[61] S. H. Payne, A. Wierzbicki, and H. J. Kreuzer, Surf. Sci. 291, 242 (1993).

[62] D. ben-Avraham and J. Köhler, Phys. Rev. A 45, 8358 (1992).

[63] J. W. Evans, D. K. Hoffman, and H. Pak, Surf. Sci. 192, 475 (1987).

[64] J. L. Sales and G. Zgrablich, Phys. Rev. B 35, 9520 (1987); Surf. Sci. 187, 1 (1987).

[65] J. L. Sales, R. O. Uñac, M. V. Gargiulo, V. Bustos, and G. Zgrablich, Langmuir 12, 95 (1996).

[66] C. Stampfl, H. J. Kreuzer, S. H. Payne, H. Pfnür, and M. Scheffler, Phys. Rev. Lett. 83, 2993 (1999).

[67] E. Hansen and M. Neurock, Surf. Sci. 441, 410 (1999).

[68] J.-S. McEwen and A. Eichler, J. Chem. Phys. 126, 094701 (2007).

[69] A. P. J. Jansen and W. K. Offermans, J. Comp. Meth. Sci. 2, 351 (2002).

[70] C. G. M. Hermse, F. Frechard, A. P. van Bavel, J. J. Lukkien, J. W. Niemantsverdriet, R. A. van Santen, and A. P. J. Jansen, J. Chem. Phys. 118, 7081 (2003).

[71] V. P. Zhdanov and T. Matsushima, Phys. Rev. Lett. 98, 036101 (2007).

[72] R. Kose, W. A. Brown, and D. A. King, J. Phys. Chem. B 103, 8722 (1999).

[73] P. Piercy, K. De’Bell, and H. Pfnür, Phys. Rev. B 45, 1869 (1992).

[74] C. Schwennicke and H. Pfnür, Phys. Rev. B 56, 10558 (1997).

[75] H. Schlichting, D. Menzel, T. Brunner, W. Brenig, and J. C. Tully, Phys. Rev. Lett. 60, 2515 (1988).

[76] H. Schlichting, D. Menzel, T. Brunner, and W. Brenig, J. Chem. Phys. 97, 4453 (1992).

[77] M. Head-Gordon, J. C. Tully, H. Schlichting, and D. Menzel, J. Chem. Phys. 95, 9266 (1991).

[78] L. V. Lutsevich, O. A. Tkackenko, and V. P. Zhdanov, Langmuir 7, 1225 (1991).

[79] V. P. Zhdanov and P. R. Norton, Surf. Sci. 459, 245 (2000).

[80] A. P. J. Jansen and W. K. Offermans, Lect. Notes Comput. Sci. 3480, 1020 (2005).

[81] V. P. Zhdanov and T. Matsushima, Surf. Sci. 583, 253 (2005).

[82] A. Hellman and K. Honkala, J. Chem. Phys. 127, 194704 (2007).

[83] R. A. van Santen and J. W. Niemantsverdriet, Chemical Kinetics and Catalysis (Plenum Press, New York, 1995). 